\title{
Validación de un cuestionario de satisfacción con el servicio de fisioterapia en residencias de mayores institucionalizados Validation of a satisfaction questionnaire with physiotherapy service in institutionalized nursing homes
}

\author{
César Raúl Rodríguez-Martín ${ }^{1}$. Remedios López-Liria², David Padilla-Góngora ${ }^{3}$ \\ ${ }^{1}$ Centro de Personas Mayores "Bartolomé Sánchez Moreno" - Oria, Almería \\ 2 Facultad de Ciencias de la Salud. Universidad de Almería \\ ${ }^{3}$ Facultad de Psicología. Universidad de Almería
}

Fecha de recepción: 12/12/2015 - Fecha de aceptación: 23/05/2016

\begin{abstract}
Resumen
Introducción. La progresiva incorporación del fisioterapeuta como parte del equipo multidisciplinar en las instituciones geriátricas de todo el país se ha hecho evidente. Pero los datos sobre el grado de satisfacción que los usuarios tienen con este servicio son casi inexistentes, debido a la falta de instrumentos fiables que lo midan. Objetivo. Diseñar y validar un cuestionario útil que defina la satisfacción que tienen los usuarios de centros geriátricos con el servicio de fisioterapia que reciben. Metodología. Se establecieron diversos bloques temáticos para componer las principales cuestiones a valorar en la función del fisioterapeuta dentro de una residencia, información que fue sometida a consulta en un panel de expertos. Tras el análisis realizado por estos jueces-expertos, se generó a través de consenso una serie de ítems que conformarían el cuestionario provisional sobre satisfacción con el servicio de fisioterapia teniendo en cuenta la validez de contenido, constructo, claridad e inteligibilidad de la información. Este cuestionario provisional fue testado en una muestra de 100 usuarios; el cuestionario final se sometió a la valoración de una muestra de 353 mayores institucionalizados en Andalucía. Resultados y Conclusiones. Se ofrece un cuestionario validado que permita expresar la satisfacción con el servicio de fisioterapia en las residencias de mayores. La información que este cuestionario puede generar, contribuiría a desvelar cuales son las actuales fortalezas y debilidades de los servicios de fisioterapia prestados, abriendo nuevos horizontes tanto para la investigación como para la gestión y mejora de los mismos.

Palabras clave: Cuestionario, Satisfacción, Mayores, Institución, Fisioterapia
\end{abstract}

\begin{abstract}
Introduction. The progressive incorporation of the physiotherapist as part of the multidisciplinary team in geriatric institutions around the country has become evident. But data on the degree of satisfaction of users with this service are almost non-existent, due to the lack of reliable instruments for measuring it. Objective. Design and validate a useful questionnaire which defines the satisfaction of users of geriatric centers with the physiotherapy service they receive. Methodology. Different thematic blocks were established to compose the main issues in the role of the physiotherapist within a residence, information that was submitted to a panel of expert's consultation. Following the analysis carried out by these judges-experts, generated through consensus a series of items that would form the provisional questionnaire on satisfaction with the physiotherapy service taking into account the validity of content, construct, clarity and intelligibility of information. This provisional questionnaire was tested on a sample of 100 users; the final questionnaire was submitted to the assessment of a sample of 353 institutionalized elders in Andalusia. Results and conclusions. Provides a validated questionnaire that allows expressing satisfaction with the physiotherapy service in nursing homes. The information that this questionnaire can generate could contribute to reveal what are the current strengths and weaknesses of the physiotherapy services provided and opening new horizons both for research and for the management and improvement of them. Keywords: Questionnaire, Satisfaction, Elders, Institution, Physiotherapy.
\end{abstract}

\footnotetext{
*Correspondencia: crrm 9@hotmail.com
} 


\section{Introducción}

La atención a las personas en situación de dependencia y la promoción de su autonomía constituye uno de los principales retos de la política social en los países de Europa. Reto que consiste en atender las necesidades de aquellos, que por encontrarse en situación de especial vulnerabilidad, requieren apoyos para desarrollar las actividades básicas de la vida diaria, alcanzar una mayor autonomía personal y poder ejercer plenamente sus derechos de ciudadanía.

La Ley de la Dependencia se proponía establecer en España un nuevo derecho universal y subjetivo de ciudadanía que garantizara un cierto nivel de atención y cuidados a cualquier persona dependiente (mayores y personas con discapacidad que no se pudiesen valer por sí mismas). A partir de su entrada en vigor, el 1 de enero de 2007, todos los españoles que lo solicitaron comenzaron a ser evaluados para determinar su grado y nivel de dependencia y las prestaciones a las que tendría derecho. Encontrándose un amplio catálogo de servicios sociales que incluiría los centros de día y los residenciales para mayores dependientes, entre otros (Boletín Oficial del Estado, 2006).

Las unidades de estancia diurna o nocturna, son centros de promoción de bienestar de las personas mayores para al fomento de la convivencia, integración, participación, solidaridad y la relación con el medio social, pudiendo servir de apoyo para la prestación de servicios sociales a otros sectores de la población. Ofrecen actividades culturales, turísticas, recreativas, artesanales, de autoayuda y servicios de rehabilitación, comedor, cafetería, etc. Su principal objetivo es mejorar o/y apoyar el grado de autonomía de los usuarios y las familias o cuidadores que los atienden (Instituto de Mayores y Servicios Sociales, 2000).

En el ámbito de las instituciones geriátricas, la incorporación del fisioterapeuta como parte del equipo multidisciplinar está siendo cada vez mayor. Sin embargo, existen escasos estudios que definan con claridad sus funciones y menos aún la valoración que hacen los usuarios de los servicios que reciben.

Las encuestas de satisfacción de residentes

Uno de los objetivos del análisis de la satisfacción es el de facilitar información a los profesionales, gestores y administradores de residencias geriátricas sobre aquellos aspectos de la organización que son percibidos por los residentes como insatisfactorios y que son susceptibles de mejora mediante la modificación de circunstancias, comportamientos o actitudes.

Se ha señalado que la metodología más recomendable para valorar esta satisfacción es la que combina técnicas de investigación cualitativa y cuantitativa, así como el empleo de instrumentos que faciliten la evaluación de la percepción de los residentes sobre si determinados hechos de buena praxis han ocurrido. La interpretación y el establecimiento de planes de mejora se relacionan con la 
utilidad de los cuestionarios de satisfacción elaborados hasta el momento (Quinn, Jacobsen, Albrecht, Bell, Newman, Bell \& Ruckdeschel, 2004). Las encuestas han sido los instrumentos más utilizados para valorar la satisfacción de los residentes, constituyendo además un componente importante de los programas de evaluación y mejora de la calidad (ISO 9001-2008, EFQM; Sánchez, 2002; Saturno, 1995; Urden, 2002).

Pero aún persiste el continuo debate sobre si las preguntas abiertas producen mayor información que las estructuradas y cuáles son las escalas de evaluación que obtienen una respuesta más crítica. Un factor común a diversos estudios de satisfacción es que muy pocos residentes expresan el descontento con la atención que reciben, por ello suelen encontrarse casi siempre niveles de satisfacción muy elevados (alrededor del $80 \%$ ) o con poca variabilidad. Aunque estos datos pueden agradar a los administradores de servicios geriátricos, constituyen un problema para los investigadores, ya que a menudo son forzados a comparar las respuestas más positivas con las menos positivas, fenómeno que se explica en parte, por el problema metodológico de cómo se hacen las preguntas o la existencia de ítems directos únicamente (Collins \& O’Cathain, 2003; Williams, Coyle \& Healy, 1998).

En el desarrollo de las encuestas puede utilizarse uno o múltiples ítems que respondan a la cuestión sobre satisfacción. Se ha descrito que las evaluaciones de un sólo ítem ofrecen simplicidad y velocidad para los propósitos de hacer una auditoría, reduciendo la carga del residente cuando se necesita determinar también otros resultados. No obstante, estas evaluaciones se toman casi siempre con desconfianza, ya que sus propiedades psicométricas son discutibles. En cambio, las evaluaciones con múltiples preguntas pueden incluir varios ítems que contribuyan en conjunto a una variable, o varias subescalas que midan dimensiones diferentes de satisfacción (Caminal, 2001; Herborg, 2001; Maceiras, 2002; Otani, Kurz \& Harris, 2005; Saturno, 1995; Speight, 2005; Sitzia \& Wood, 1997; Solas \& Silio, 2000; Urden, 2002; Williams et al., 1998).

Entre las cuestiones incluidas en los cuestionarios de satisfacción se han hallado preguntas relacionadas con los resultados del tratamiento (atención interpersonal, cuidado clínico, accesibilidad, etc.), ítems que se refieren a la experiencia (el proceso), a los resultados, preguntas que se relacionan con la predicción de la satisfacción (expectativas) o ítems que se vinculan a las consecuencias de la satisfacción (como la intención de continuar con el tratamiento) (Caminal, 2001; Herborg, 2001; Maceiras, 2002; Otani, Kurz \& Harris, 2005; Saturno, 1995; Sitzia \& Wood, 1997; Speight, 2005; Urden, 2002; Williams et al., 1998).

Para este trabajo, se han utilizado tres de las conceptualizaciones de evaluación sobre satisfacción descritas por Schommer, Wenzel \& Kukukarsian (2002). La primera, denominada "satisfacción como evaluación de desempeño", pretende valorar la competencia del personal en la prestación del servicio. Considerando que este bloque podría limitar la oportunidad de los residentes a manifestar sus opiniones, se dejó un apartado final abierto a efectos de que se pudiesen expresar comentarios 
adicionales. La segunda conceptualización fue la de "satisfacción como evaluación de auto-eficacia", para abordar mayoritariamente los resultados de la prestación del servicio en las residencias geriátricas. La tercera fue la de "satisfacción como una valoración basada en los afectos", dado que también se le demandó al residente la respuesta emocional que recibió de los diferentes profesionales en la realización de su labor diaria (Ramos, Martínez-Tur, Tordera \& Peiró, 2000).

\section{Objetivo}

Diseñar y validar un cuestionario útil para conocer la satisfacción con el servicio de fisioterapia que tienen los usuarios de centros geriátricos.

\section{Material y método}

Investigación de tipo instrumental que ha consistido en la realización de las fases necesarias para la validación de un cuestionario según Montero y León (2001) o Carretero-Dios y Pérez (2005).

La generación de los ítems se realizó tomando como base varios documentos como los Convenios Colectivos para Residencias de Personas Mayores y las normas UNE de certificación de calidad, donde se definen las características más importantes del servicio de fisioterapia. Se realizó, además, una búsqueda bibliográfica sobre publicaciones relacionadas, con el objetivo de valorar el contenido de los ítems a incluir (Asociación Española de Normalización y Certificación, AENOR, 2000; Boletín Oficial del Estado, 2008).

Para la elaboración de las respuestas a cada ítem se utilizó una escala de valoración aditiva (tipo Likert), que es la más utilizada para evaluar actitudes y percepciones. Es decir, cada ítem fue presentado como una declaración afirmativa seguida de diversas opciones de respuesta graduales (nada de acuerdo, poco de acuerdo, de acuerdo, bastante de acuerdo y muy de acuerdo) asignándoles una puntuación (de 0 a 4). La puntuación total de cada encuestado se podría obtener sumando la calificación de cada ítem (Rodríguez-Martín, 2011). Existiendo un apartado final que ofrecería la posibilidad de realizar alguna observación que matizara las respuestas.

Se prestó especial atención al formato del instrumento y para su diseño se tuvo en cuenta diagramar adecuadamente, dejar espacios suficientes para las respuestas abiertas, resaltar las opciones de respuesta múltiple y evitar preguntas de clasificación (que son aquellas en las que los residentes deben responder a distintas preguntas dependiendo de sus respuestas anteriores). El cuestionario ocupó dos páginas, encabezado por una explicación de cómo rellenar el mismo y el significado de las diferentes puntuaciones. En éste se puede observar que aparecen preguntas de diversa índole, unas referidas al aspecto humano de la relación laboral y otras a la profesionalidad, dado que se ha 
justificado previamente que en la satisfacción del usuario influye tanto el aspecto laboral como el social.

El cuestionario provisional se envió a 10 expertos para asegurar la representatividad y la generalidad de los resultados que pudiesen derivarse del mismo. Dicho panel estuvo compuesto por profesionales que habían prestado servicios con más de 10 años de experiencia en instituciones residenciales para mayores de la provincia de Almería.

Los cuestionarios (enviados vía electrónica, correo postal y entregados en mano) se rellenaron de forma anónima y la revisión se efectuó en tres rondas, aplicando la metodología Delphi.

La revisión se llevó a cabo de una manera anónima para evitar los efectos de "líderes" (Astigarraga, 2003). Entre las principales características del método Delphi se encuentra el garantizar la confidencialidad en las opiniones de cada uno de los integrantes, no realizar intercambios de opinión entre los diferentes panelistas y se especializa por la búsqueda de consenso entre los participantes (Astigarraga, 2003). Este objetivo se consigue mediante la realización de más de una ronda a fin de proponer a los expertos un feedback de la opinión del panel. En cada ronda se envió a los expertos una carta explicándoles la finalidad del cuestionario y los objetivos que se perseguían con su desarrollo; adjuntándoles además, los sucesivos cuestionarios propuestos.

En la primera ronda se envió un listado de 20 posibles ítems a cada profesional, teniendo en cuenta que tras los sucesivos análisis podrían descartarse más de uno. Las opiniones de los expertos evaluaron la validez de contenido y aseguraron la relevancia y la claridad de los ítems propuestos. Para la modificación, exclusión o inclusión de ítems se tuvo en cuenta el acuerdo obtenido por la mayoría de los expertos. A partir de las opiniones obtenidas en la primera ronda, se reformuló el cuestionario conteniendo una nueva propuesta de ítems a incluir, que fueron sometidos nuevamente a las opiniones de los expertos, depurando aquellos con las puntuaciones más bajas y seleccionando finalmente diez que reunieron los valores mayores (Galíndez \& Casas, 2011). Finalmente tuvo lugar una reunión presencial entre los expertos y los investigadores donde se pudieron acordar más aspectos cruciales del instrumento de medida.

A partir del cuestionario resultante de la revisión por el panel de expertos, se realizó un pilotaje a 20 usuarios de una residencia geriátrica de la provincia de Almería con el fin de evaluar la comprensión de los ítems por los mayores y la utilidad de la escala de satisfacción, eliminando o reformulando aquellas preguntas difíciles de entender o responder. Durante este pilotaje, también se valoró el tiempo necesario para completar el cuestionario y la impresión de los residentes sobre la facilidad de calificación de la escala Likert empleada, además de sus comentarios adicionales.

Una vez confeccionado el cuestionario provisional, con esta serie de modificaciones, la siguiente fase consistiría en la aplicación del mismo en una muestra de 100 residentes. De cara a la validación 
estadística de este cuestionario piloto y buscando que la muestra fuese representativa, se seleccionaron residentes de centros públicos, concertados y privados de la Comunidad Autónoma de Andalucía. Contactando en primer lugar con el director de cada centro, al que se le explicó la investigación con objeto de solicitar su colaboración y acordar un horario adecuado donde pudieran participar las personas mayores.

A través del mismo procedimiento, la validación final del Cuestionario de Satisfacción definitivo se logró en una muestra de 353 residentes de todas las edades procedentes de centros geriátricos públicos, concertados y privados de la población geriátrica en Andalucía (Carretero-Dios, 2005 \& Martínez-Arias, 1995).

\section{Resultados}

A partir de los datos obtenidos de los 353 residentes se realizaron los siguientes análisis estadísticos de cara a la validación del cuestionario definitivo (ver anexo I):

Análisis de la calidad técnica de los ítems y fiabilidad

En primer lugar se valoró la comprensión de los ítems mediante el criterio de los residentes que señalaron aquellos ítems cuyo significado no estaba totalmente claro. Posteriormente se calculó el cociente de variación para cada uno de los ítems (tabla I), los valores marcados en negrita son aquellos cuyo cociente de variación fue superior al considerado normal (entre el 20-40\%). Un valor elevado indica una variabilidad excesiva en las respuestas de los residentes a cada uno de los ítems.

\begin{tabular}{|c|c|c|c|c|}
\hline \multicolumn{5}{|c|}{ ESTADÍSTICOS DE LOS ELEMENTOS } \\
\hline & $\mathbf{N}$ & Media & Desv.Típica & C. Variación \\
\hline Item 1 & 353 & 2,65 & 0,57 & 21,66 \\
\hline Item 2 & 353 & 2,64 & 0,57 & 21,66 \\
\hline Item 3 & 353 & 2,76 & 0,45 & 16,34 \\
\hline Item 4 & 353 & 2,69 & 0,50 & 18,73 \\
\hline Item 5 & 353 & 2,67 & 0,55 & 20,74 \\
\hline Item 6 & 353 & 2,68 & 0,54 & 20,14 \\
\hline Item 7 & 353 & 2,60 & 0,58 & 22,34 \\
\hline Item 8 & 353 & 2,58 & 0,59 & 22,98 \\
\hline Item 9 & 353 & 2,61 & 0,57 & 21,83 \\
\hline Item 10 & 353 & 2,58 & 0,57 & 22,24 \\
\hline
\end{tabular}

Tabla I. Estadísticos descriptivos de los elementos. Cuestionario definitivo. 
Se analizó la fiabilidad del cuestionario, a través del coeficiente de consistencia interna Alpha de Cronbach $(0,932)$.

\section{Validez de Constructo}

Se calculó mediante análisis factorial con rotación varimax, eliminando aquellos ítems con saturaciones bajas en algún factor $(<0.30)$ o los que lo hacían en más de un factor a la vez (Santiesteban, 1990). Un solo factor ha explicado el 62,228 \% de la varianza en este servicio, por lo que todos los ítems se engloban en el factor, "Funciones servicio fisioterapia" (tablas II y III).

\section{TOTAL VARIANZA EXPLICADA}

\begin{tabular}{|c|c|c|c|c|c|c|}
\hline \multirow{2}{*}{ Componente } & \multicolumn{3}{|c|}{ Autovalores iniciales } & \multicolumn{3}{|c|}{$\begin{array}{l}\text { Suma de las saturaciones al } \\
\text { cuadrado de la extracción }\end{array}$} \\
\hline & Total & $\begin{array}{c}\% \text { de } \\
\text { Varianza }\end{array}$ & $\begin{array}{c}\% \\
\text { Acumulado }\end{array}$ & Total & $\begin{array}{c}\% \text { de } \\
\text { Varianza }\end{array}$ & $\begin{array}{c}\% \\
\text { Acumulado }\end{array}$ \\
\hline 1 & 6,223 & 62,228 & 62,228 & 6,223 & 62,228 & 62,228 \\
\hline 2 & 690 & 6,902 & 69,130 & & & \\
\hline 3 &, 536 & 5,356 & 74,486 & & & \\
\hline 4 &, 527 & 5,275 & 79,761 & & & \\
\hline 5 &, 503 & 5,028 & 84,788 & & & \\
\hline 6 & ,393 & 3,931 & 88,719 & & & \\
\hline 7 & ,351 & 3,507 & 92,226 & & & \\
\hline 8 & ,294 & 2,944 & 95,169 & & & \\
\hline 9 & ,286 & 2,863 & 98,032 & & & \\
\hline 10 & ,197 & 1,968 & 100,000 & & & \\
\hline
\end{tabular}

Método de Extracción: Análisis de Componentes Principales.

Tabla II. Total Varianza Explicada. Análisis factorial. Servicio de fisioterapia.

\section{Discusión}

Debido a la evidente necesidad de evaluar los servicios de fisioterapia en las residencias de mayores, en este estudio se han realizado los pasos que son necesarios para crear un instrumento que mida la satisfacción de estas personas, contando en primer lugar con las aportaciones de expertos en la materia y la posterior colaboración de los residentes como población diana para el pilotaje de dicho cuestionario. Este trabajo se ha desarrollado a través de la construcción de un cuestionario provisional basado en la literatura científica existente, sobre el que se han realizado cambios y mejoras basados en la opinión de expertos y la propia población a la que va a estar dirigido, para finalmente constituir un cuestionario que sea válido, representativo y que se dirija a muestras de 
personas mayores, con el deseo de que pueda ser un instrumento de evaluación útil en la mejora de la calidad del servicio de fisioterapia en residencias.

\begin{tabular}{cc}
\hline \multicolumn{3}{c}{ MATRIZ DE COMPONENTES $^{\text {a }}$} \\
& Componente \\
1.Tiempo entre ingreso e inicio tratamiento & 1 \\
2. Información objetivos y tratamiento &, 739 \\
3. Trato amable y respetuoso &, 727 \\
4. Respeto privacidad e intimidad &, 768 \\
5. Opinión y participación en tratamiento &, 836 \\
6. Clima de trabajo individual y grupal &, 843 \\
7. Adaptación horario tratamiento &, 797 \\
8. Variedad y disponibilidad material &, 772 \\
9. Información progresos tratamiento &, 834 \\
10. Participación alta tratamiento &, 783 \\
Método de Extracción: Análisis de Componentes Principales.
\end{tabular}

a. 1 componente extraído.

Tabla III. Matriz de componentes. Servicio de fisioterapia.

En la literatura científica existente hoy en día, se han hallado algunos estudios de satisfacción publicados, los más tempranos partieron de la identificación de las características de los residentes como la edad, género o raza, con el objetivo de predecir los niveles de satisfacción (Caminal, 2001; Saturno, 1995; Urden, 2002). Otros trabajos han investigado los atributos de la atención médica, de enfermería, los procesos de admisión, etc., con el propósito de identificar las propiedades que influyen en la satisfacción global de los residentes (Sitzia \& Wood, 1997; Yellen, Davis \& Ricard, 2002). Por otro lado, también se han analizado las propiedades psicométricas de algunos instrumentos de valoración de la satisfacción, estudiado la relación entre los servicios de las residencias geriátricas y la satisfacción global del residente (Asadi-Lari, Tamburini \& Gray, 2004; Herborg, 2001; Rider, 2002; Quinn et al., 2004). Pero hasta el momento, no se han hallado investigaciones previas centradas en la satisfacción del residente con el servicio de fisioterapia.

Otro interesante estudio que se llevó a cabo en el estado de Minesota fue la "Encuesta de Satisfacción del Residente" que alcanzó un rango de fiabilidad de 0,53-0,77, basándose en 52 ítems relacionados con categorías referentes a la evaluación general del centro, las actividades, la adaptación del entorno, la alimentación, las relaciones con los trabajadores, la seguridad, privacidad, autonomía, etc. (Kane, Kling, Bershadsky, Giles, Degenholtz, Liu et al., 2003). En algunos países de Europa se suelen utilizar cuestionarios propuestos por el estado para valorar la satisfacción de los 
usuarios en las residencias. En Francia, este tipo de cuestionario llamado Angélique, pretende valorar las expectativas y la satisfacción de los residentes y las familias; en una segunda parte se centra en las posibilidades ofrecidas al residente en términos de autonomía, de acompañamiento, de cuidados; y finalmente evalúa las relaciones del centro con las demás estructuras (Ministére du travail, des relations sociales, de la familleet de la dolidarité, 2008).

Además, se debe señalar que diversos autores han apoyado la idea de que al evaluar la satisfacción ha de considerarse que las creencias y los valores de los residentes pueden cambiar con el tiempo, aspecto que se denomina "cambio de respuesta". Por consiguiente, una valoración apropiada de satisfacción requeriría que fuese repetida de manera periódica (Herborg, 2001; Caminal, 2001; Maceiras, 2002; Otani, Kurz \& Harris, 2005; Saturno Hernández, 1995; Solas Gaspar \& Silio Villamil, 2000; Urden, 2002).

\section{Conclusión}

Tras la consulta realizada a los expertos en fisioterapia se ha obtenido una serie de ítems que han ayudado a crear un cuestionario útil que ha sido validado estadísticamente para que las personas mayores puedan expresar su satisfacción con este servicio.

La información que este cuestionario puede generar tras su utilización, podría contribuir a desvelar cuales son las actuales fortalezas y debilidades del servicio de fisioterapia prestado en cada uno de los centros de mayores, abriendo nuevos horizontes tanto para la investigación como para la gestión y mejora de los mismos.

\section{Bibliografía}

Asadi-Lare, M., Tamburini, M. \& Gray, D. (2004). Patients'needs, satisfaction, and heath related quality of life: Towards a comprehensive model. Health and Quality of life Outcomes, 2, 32. doi: 10.1186/1477-7525-2-32

Asociación Española de Normalización y Certificación (AENOR, 2000). Norma UNE 158001 de Gestión de Servicios en las residencias de mayores. Madrid: AENOR.

Astigarraga, E. (2003). Método Delphi. Universidad de Deusto. Recuperado de http: www.echalemojo.com.

Boletín Oficial del Estado (BOE, 2006). Ley 39/2006, de 14 de diciembre, de promoción de la Autonomía Personal y Atención a las personas en situación de dependencia. Boletín Oficial del Estado, 299, 44142-44156.

Boletín Oficial del Estado (BOE, 2008). Resolución de 26 de marzo de 2008, de la Dirección General de Trabajo, por la que se registra y publica el $V$ Convenio colectivo marco estatal de servicios de 
atención a las personas dependientes y desarrollo de la promoción de la autonomía personal. Boletín Oficial del Estado, 79, 18281-18305.

Caminal, J. (2001). La medida de la satisfacción: un instrumento de participación de la población en la mejora de la calidad de los servicios sanitarios. Revista de Calidad Asistencial, 16, 276-279.

Carretero-Dios, H., \& Pérez, C. (2005). Normas para el desarrollo y revisión de estudios instrumentales. [Standards development and revision of instrumental studies]. International Journal of Clinical and Health Psichology, 5(3), 521-551.

Collins, K., \& O'Cathain, A. (2003). The continuum of patient satisfaction -from satisfied to very satisfied. Social Science Medicine, 57, 2465-70. doi: 10.1016\%2FS0277-9536\%2803\%29000984

Galíndez, E., \& Casas, F. (2011). Adaptación y validación de la MSLSS de satisfacción vital multidimensional con una muestra de adolescentes. Revista de Psicología Social, 26(3), 309323. doi: $10.1174 \% 2 F 021347411797361284$

Herborg, H. (2001). Satisfaction-How can we measure change? Quality Issues Working Conference PCNE 2001

Instituto de Mayores y Servicios Sociales (IMSERSO, 2000). Las personas mayores en España. Informe 2000. [Older people in Spain: Report 2000]. Madrid: IMSERSO.

Kane, R. A., Kling, K. C., Bershadsky, B., Kane, R. L., Giles, K., Degenholtz, H. B., Liu, J. \& Cutler, L. J. (2003). Quality of Life Measures for Nursing Home Residents. Journal of Gerontology: Medical Sciences, 58A (3), 240-248.

Maceiras, L. (2002). Encuestas de satisfacción de usuarios. [User satisfaction surveys]. Salud Pública Educación Salud, 2(1), 28-33.

Ministère du travail, des relations socials, de la famille et de la solidarité. (2008). Gestion des risques del maltraitance en establissement. En http://travailemploi.gouv.fr/IMG/pdf/guide_gestion_des_risquesPAPHetab_2_.pdf

Montero, I., \& León, O. G. (2001). Usos y costumbres metodológicos en la Psicología española: un análisis a través de la vida de psicothema (1990-1999). [Methodological practices and customs in Spanish psychology: an analysis through the life of Psicothema (1990-1999).]. Psicothema, 13, 671-677.

Otani, K., Kurz, R., \& Harris, L. (2005). Managing primary care using patient satisfaction measures. Journal of healthcare Management, 50(5), 311-24.

Quinn, G., Jacobsen, P., Albrecht, T., Bell, B., Newman, L., Bell, M., \& Ruckdeschel, J. (2004). Real time patient satisfaction survey and improvement process. Hospital Topics, 82(3), 26-32. doi:10.3200\%2FHTPS.82.3.26-32

Ramos, J., Martínez-Tur, V., Tordera, M., \& Peiró, J. (2000). Contribuciones de la Psicología Social al estudio de la satisfacción de los usuarios y consumidores. [Contributions of social psychology to the study of user satisfaction and consumer]. Revista de Psicología Social, 15(2), 5-24. doi:10.1174/021347400760259776

Rider, E. (2002). Performance profiles: the influence of patient satisfaction data on physicians' practice. Pediatrics, 109(5), 752-7. 
Rodríguez-Martín, C. R. (Abril 2011). Diseño de un cuestionario de satisfacción de los usuarios con los servicios de las residencias de mayores. Memoria Docente e investigadora del Programa de Doctorado Gerontología y Geriatría. [Designing a questionnaire of user satisfaction with the services of nursing homes. Teacher and researcher Memory Program Doctoral Gerontology and Geriatrics.]. Universidad de Almería.

Sánchez, E. (2002). Modelo EFQM y calidad asistencial. [EFQM model and quality of care]. Revista de Calidad Asistencial, 17(2), 64-66.

Saturno, P. (1995). Los métodos de participación del usuario en la evaluación y mejora de la calidad de los servicios sanitarios. [Methods of user involvement in the evaluation and improvement of the quality of health services.]. Revista Española Salud Pública, 69, 163-175.

Schommer, J., Wenzel, R., \& Kukukarsian, S. (2002). Evaluation of pharmacists services for hospital inpatients. American Journal of Health-System Pharmacy, 59(1), 1632-37.

Sitzia, J., \& Wood, N. (1997). Patient Satisfaction: A review of issues and concepts. Social Science Medicine, 45(12), 1829-1843.

Solas, O., \& Silio, F. (2000). Los retos directivos ante la gestión de la efectividad. [The challenges managers to management effectiveness.]. Granada: Escuela Andaluza de Salud Pública.

Speight, J. (2005). Assessing patient satisfaction: Concepts, Applications and Measurement. Value in Health, 8(1), 56-8. doi: 10.1111\%2Fj.1524-4733.2005.00071.x

Urden, L. (2002). Patient Satisfaction measurement: current issues and implications. Outcomes Management, 6 (3), 125-131.

Williams, B., Coyle, J., \& Healy, D. (1998). The meaning of patient satisfaction: an explanation of high reported levels. Social Science Medicine, 47(9), 1351-59. doi: 10.1016/S0277-9536(98)00213-5

Yellen, E., Davis, G., \& Ricard, R. (2002). The measurement of patient satisfaction. Journal of Nursing Care Quality, 16(4), 23-9. doi: 10.1097\%2F00001786-200207000-00005 


\section{Anexo}

Modelo final cuestionario de satisfacción para el servicio de fisioterapia

SEXO:

EDAD:

FECHA DE INGRESO:

\section{FECHA REALIZACIÓN CUESTIONARIO:}

INSTRUCCIONES. Usted ha sido seleccionado como usuario de una residencia geriátrica, para participar en un estudio sobre la satisfacción con el servicio de fisioterapia recibido. Con sus respuestas, que serán tratadas de forma anónima, se espera que contribuyan a evaluar la calidad de esta atención y a realizar orientaciones para su mantenimiento o mejora.

Por favor, valore cada pregunta ofreciendo una puntuación numérica según la escala adjunta de 0 a 3 (que puntúa gradualmente la satisfacción de nada a muy satisfecho). Asimismo, si lo considera oportuno, puede realizar comentarios en el campo reservado a observaciones.
0
1
2
3
Nada satisfecho
|-----------------|-------------------|-------------------|
Poco satisfecho
Satisfecho
Muy satisfecho

\section{SERVICIO DE FISIOTERAPIA}

NOMBRE DE REFERENCIA:

1- ¿Está usted satisfecho con el tiempo que transcurrió entre el ingreso en el centro y el inicio del tratamiento de Fisioterapia?

Observaciones:

2- ¿Se siente usted satisfecho con la información que le da el fisioterapeuta sobre los diferentes objetivos y técnicas de tratamiento que le realiza?

Observaciones:

3- ¿Se siente satisfecho con el trato amable y respetuoso con que le trata el fisioterapeuta?

Observaciones:

4- ¿Está usted satisfecho/a con el respeto a la privacidad e intimidad con que recibe el tratamiento del fisioterapeuta?

Observaciones:

5- ¿Qué opina en relación a que el fisioterapeuta le escuche y le deje participar en su tratamiento?

Observaciones:

6- ¿Está usted satisfecho con el clima de trabajo que se crea en el tratamiento individual o grupal de fisioterapia? 


\section{Observaciones:}

7- ¿La adaptación del tratamiento de fisioterapia al horario que mejor le conviene influye en su satisfacción?

Observaciones:

8- ¿Se siente usted satisfecho con la variedad de material de fisioterapia de que dispone?

\section{Observaciones:}

9- ¿Qué le parece la información que le da el fisioterapeuta sobre los progresos que tiene en su tratamiento?

Observaciones:

10- ¿Está usted satisfecho con la posibilidad de que el fisioterapeuta le deje participar en el alta de su tratamiento?

Observaciones: 